\title{
INTERACTIONS, MERGERS, AND QSO ACTIVITY
}

\author{
ALAN STOCKTON \\ Institute for Astronomy, University of Hawaii \\ 2680 Woodlawn Drive, Honolulu, Hawaii 96822
}

\section{Introduction}

Is QSO activity sometimes triggered by interactions or mergers of galaxies? Are all QSOs the result of interactions or mergers? Such questions, arising explicitly in the late 1970s, had their roots in the well-known Stoking the Furnace? section of Toomre \& Toomre (1972), the Feeding the Monster paper of Gunn (1979), and the early observational evidence that weaker forms of nuclear activity, such as Seyferts, seemed often to be found in interacting systems (e.g., Adams 1977). Reviews of the evidence regarding the relation of interactions and mergers to QSO activity have been given by Stockton (1990) and Heckman (1990). Some of the more important developments since these reviews are:

(1.) Modeling the gaseous component in $N$-body simulations - That strong interactions and mergers might "bring deep into a galaxy a fairly sudden supply of fresh fuel" (Toomre \& Toomre 1972) has long seemed a plausible, but untested, speculation. The inclusion of a gaseous component in N-body simulations of mergers (Barnes \& Hernquist 1991, 1992) has shown that much of the gas does, indeed, settle in towards the center of the merger remnant, although the models still fall short by about a factor of $10^{7}$ of having the resolution range to follow gas from galactic scales to the scale of a supermassive black hole accretion disk.

(2.) IR imaging of QSO hosts - The advent of low-noise IR-array detectors of increasing size has led to a number of programs of IR imaging of QSO host galaxies (e.g., Dunlop et al. 1993; McLeod \& Rieke 1994). The scientific driver for these surveys is shown in Fig. 1 (see also McLeod \& Rieke 1995): for QSOs with $z \sim 0.3$, observations in the $\mathrm{J}$ or $\mathrm{H}$ bands sample close to the peak in the spectral-energy distribution of stellar populations with ages $\gtrsim 1 \mathrm{Gyr}$ as well as close to where such populations show greatest contrast with respect to the QSO nuclear light. This ability to concentrate on the 


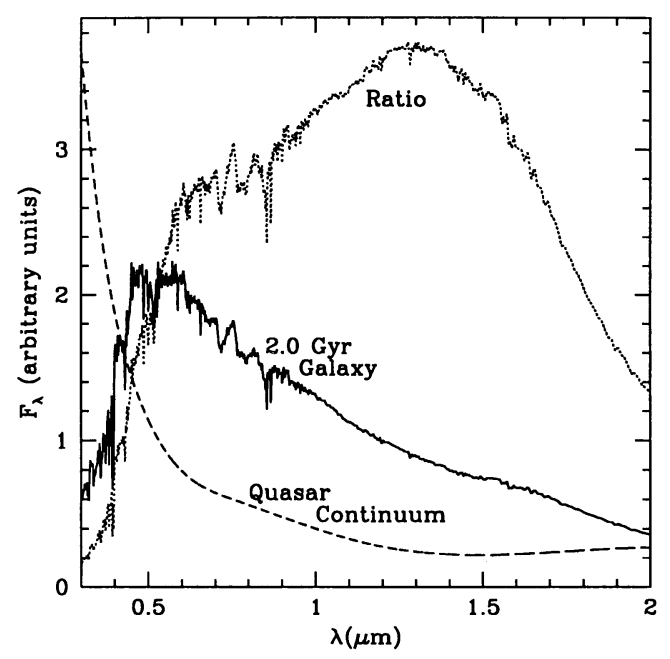

Figure 1. The advantage of near-IR observations for detecting older stellar populations in QSO host galaxies. The solid curve is a Bruzual-Charlot (1997) model for a 2-Gyr-old stellar population, the dashed curve is a median quasar continuum (Elvis et al. 1994), and the dotted curve shows the ratio of the two. At a modest redshift $(z \sim 0.3)$, the best contrast with the QSO nucleus is achieved in the $J$ and $H$ bands.

older stellar populations, normally representing most of the luminous mass of the host galaxy, opens up many possibilities for carrying out statistical tests of hypotheses regarding QSO host-galaxy formation and evolution.

(3.) HST imaging-After a flurry of controversy, the major HST imaging programs on host galaxies of low-redshift QSOs (Bahcall et al. 1994, 1995a, b, 1996, 1997; Hutchings et al. 1994; Disney et al. 1995; McLeod \& Rieke 1995) are in general agreement, both with each other and with most previous ground-based work. The HST images confirm that virtually all radio-loud QSOs are found in galaxies with (sometimes disturbed) elliptical-like light distributions, as expected. But they also show that radioquiet QSOs often have similar light distributions, a result that went against the previous consensus, even though it had been foreshadowed in some ground-based work (e.g., Hutchings \& Neff 1992).

(4.) Unified models - To the extent that unification of quasars (i.e., radioloud QSOs) and FR II radio galaxies (Scheuer 1987; Barthel 1989; Antonucci 1993) holds at some non-trivial level, one can use radio galaxies as proxies for quasar hosts. If such a substitution is possible, one can study the inner regions of quasar hosts (at least from some lines of sight) without having to deal with the overwhelming glare of the quasar nuclear light, which in these cases is conveniently masked by the quasars' own internal 
occulting "disks."

While rigorous proof of the role of strong interactions in triggering QSO activity remains elusive, the circumstantial evidence in favor of this picture is now fairly compelling, and much of the focus of current research in this area has turned instead towards attempting to trace the evolutionary history of interactions/mergers that may result in QSOs. Here I shall describe some work my colleagues and I have been doing in two specific areas: (1) the nature of "transition" objects between ultraluminous IR galaxies and the classical QSO population, and (2) the morphologies of some specific cases of host galaxies of powerful radio sources at $z \sim 1$, which may offer some insight into merger and accretion processes at early epochs.

\section{Far-Infrared Spectra and "Transition" Objects}

\subsection{INTRODUCTION}

The one definite evolutionary scenario linking mergers to QSOs that has been proposed is that of Sanders et al. $(1988 a, b)$, which suggests ultraluminous IR galaxies as the parent population of at least a significant fraction of the classical radio-quiet QSOs. For $L_{\mathrm{FIR}} \geq 10^{12} L_{\odot}$, essentially all IR-luminous galaxies show clear morphological evidence for being merging systems (the fraction of apparent mergers drops quite steeply with luminosity: at $L_{\mathrm{FIR}} \sim 5 \times 10^{10} L_{\odot}$, only about $10 \%$ show evidence for strong interaction [Sanders \& Mirabel 1996, and references therein]). Sanders et al. noted that both the bolometric luminosities and space densities of the ultraluminous objects were similar to those of radio-quiet QSOs.

Attempts to find true active nuclei in luminous IR galaxies have had varied results (Kim et al. 1995; Veilleux et al. 1995; Goldader et al. 1995; Veilleux et al. 1997; Surace et al. 1998). Most of the disagreement appears to be due to the different luminosity ranges covered by the different samples, coupled with a very steep dependence of strength of nuclear activity on IR luminosity (Kim et al. 1995; Veilleux et al. 1997).

If it were true that most or all radio-quiet QSOs follow this evolutionary path, and that the QSO luminosity is correlated with the luminosity of the IR-bright stage, then the observed correlation between IR luminosity and evidence for strong interaction would suggest that, while the more luminous QSOs may virtually all result from mergers, the fraction may be lower for lower luminosity objects. Indeed, if Seyfert galaxies are regarded as the lowluminosity continuation of the radio-quiet QSO sequence, the fact that the great majority of classical Seyferts are recognizably spiral galaxies indicates that most cannot have suffered roughly equal-mass mergers. On the other hand, one of the relatively few surprises emerging from HST imaging of lowredshift QSO hosts is that $\sim 70 \%$ of radio-quiet QSO hosts have elliptical- 
like surface-brightness profiles (Bahcall et al. 1997), consistent with being merger remnants. Thus, while the distinction between Seyfert galaxies and radio-quiet QSOs is often thought to be an arbitrary division of objects with a continuum of properties, it may actually be closely correlated with the merger status of the object.

Figure 2 shows a FIR two-color diagram, based on IRAS data from Neugebauer et al. (1986) and Sanders et al. (1988a). The classical QSOs fall in loose clump centered around the power-law line in the diagram, while the ultraluminous IR galaxies populate the lower-right corner of the diagram. I shall refer to QSOs lying close to the region occupied by the ultraluminous IR galaxies as "transition objects." Several of these are labeled in Fig. 2. That choosing such objects by their FIR spectra has physical significance is suggested by two facts: (1) The five QSOs labeled in Fig. 2 not only all show tidal tails, but they are arguably the clearest and least ambiguous examples of tidal tails among low-redshift QSOs (Stockton \& Ridgway 1991; Stockton et al. 1998b); and (2) at least three of the five are low-ionization broad absorption line (BAL) QSOs, which comprise only about $1 \%$ of opticallyselected QSOs. Voit et al. (1993) argue that low-ionization BAL QSOs are heavily dust-enshrouded objects, and Egami et al. (1996) have already made the suggestion that similar objects found at high redshifts are related to ultraluminous IR galaxies.

\subsection{WHAT IS THE NATURE OF THE "TRANSITION?"}

As I have already mentioned, considerable effort has been put into determining whether ultraluminous IR galaxies are dominated by AGN or by starbursts. But demonstration of the presence of active nuclei alone would not confirm the hypothesis that ultraluminous IR galaxies are the progenitors of QSOs: one also has to deal with the relative time scales of the physical phenomena involved. To take the extreme cases, if the QSO activity lasts much longer than the ultraluminous IR phase, then objects will move from the ultraluminous IR galaxy region to the classical QSO region in a diagram like Fig. 2, but if the nuclear activity is short-lived compared with the time it takes to clear dust from the central region, they will live out their lives as QSOs in one region of the two-color plot. In the former case, the position of an object in Fig. 2 is dominated by its stage in an evolutionary sequence; in the latter, by its intrinsic characteristics. In a realistic scenario, it is quite possible that the time scales will be roughly comparable $\left(\sim 10^{8}\right.$ years each), so the situation may well be more complicated than either of these extreme cases. 


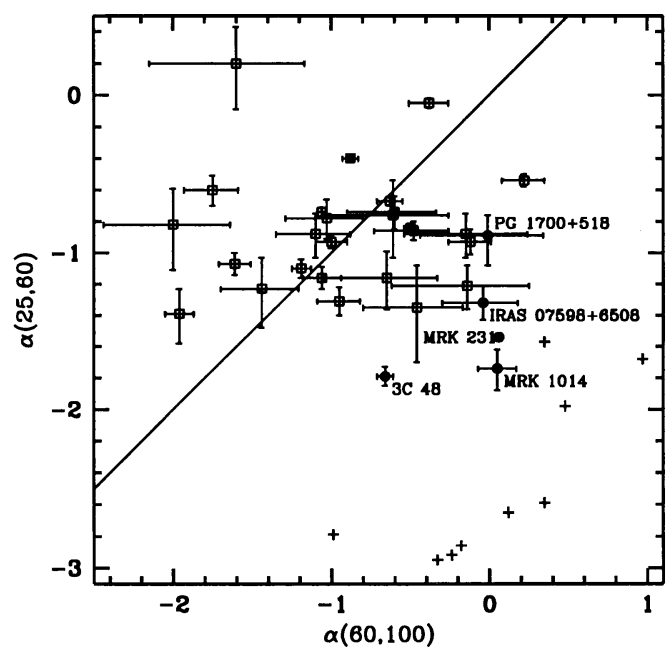

Figure 2. Far-IR two-color diagram. QSOs are generally shown as open squares with error bars, from IRAS data given by Neugebauer et al. (1986). Nine of the ten galaxies from the IRAS Bright Galaxy Survey with $L_{\text {bol }}>10^{12} L_{\odot}$ are shown as small crosses (Sanders et al. 1988a; the tenth is Mrk 231, included in the next sample). Five QSOs having colors intermediate between those of the bulk of the QSOs and the ultraluminous galaxies are indicated as closed circles and labeled. The solid diagonal line indicates the locus of power-law spectra.

\subsection{EVIDENCE THAT TRANSITION OBJECTS ARE POST-STARBURSTS}

One way to try to distinguish whether position in the FIR two-color diagram is dominated by evolution is to measure a parameter that correlates with time since the active nucleus turned on. Hutchings \& Neff (1992) have attempted to classify QSO host galaxies roughly by age since a supposed merger event, based on their optical morphologies. However, given the large range in initial parameter space covered by possible mergers and relatively small number of total resolution elements available on the host galaxies, even Hutchings \& Neff's coarse classification must suffer from some degeneracy. Moreover, the simulations of Mihos \& Hernquist (1996) indicate that the peak of the starburst activity may occur anywhere from $\sim 0$ to $\sim 5 \times 10^{8}$ years prior to the final merger, depending mostly on the internal structures of the merging galaxies. One might expect that AGN triggering would correspond more closely in time with the starburst, since both are dependent on gas flows to the inner regions.

My graduate student, Gabriela Canalizo, has been concentrating on trying to date the starbursts themselves in transition QSOs and their close 


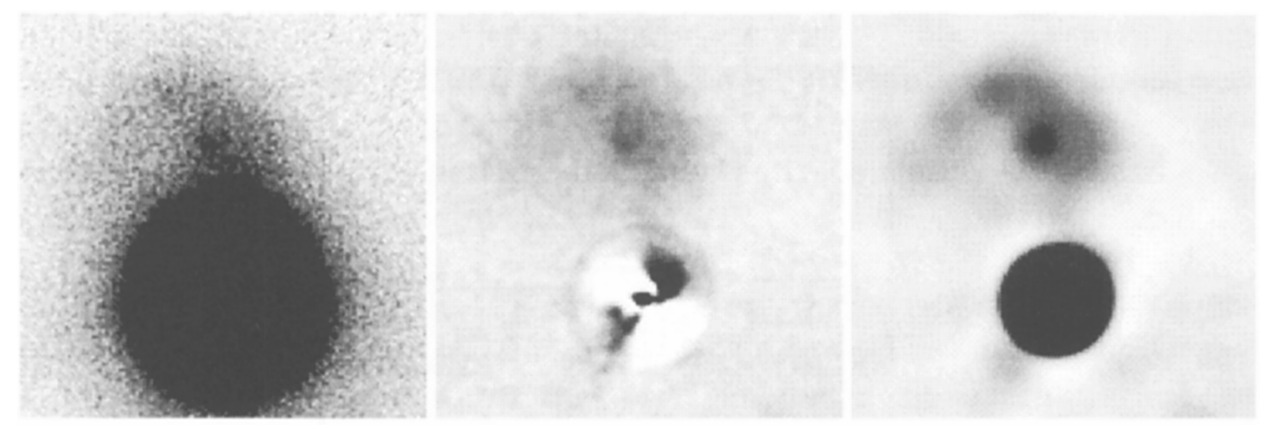

Figure 3. $\quad H$-band image of $\mathrm{PG} 1700+518$, obtained with the University of Hawaii Adaptive Optics System on the CFH Telescope. The left panel shows the recorded image, at a scale of $0^{\prime \prime} 035$ pixel $^{-1}$. The FWHM of the QSO is $0^{\prime \prime} .25$. The middle panel shows a subtraction of a model of the QSO, derived from an elliptical profile fit to the bottom half of the QSO profile, and the right panel shows a Gaussian restoration of a deconvolution of the image, using the same elliptical profile fit as the PSF kernel. Any symmetrical host galaxy is likely to be mostly removed in both the subtraction and the deconvolution. Each panel is $4^{\prime \prime} .2$ square.

companions. The idea is to estimate the time since the the most recent major starburst activity by obtaining spectra of the QSO host galaxy or companion and modeling the composite spectrum as a simple superposition of two populations: one due to the starburst and the other due to the older, pre-existing component. We have published one example, the asymmetric extension to the north of PG 1700+518 (Canalizo \& Stockton 1997). Figure 3 shows a recent adaptive-optics image (Stockton et al. 1998a) of PG $1700+518$, which shows that this feature is a close but discrete companion galaxy, with an obvious tidal tail, apparently in the process of merging with the host galaxy of PG $1700+518$ itself. We currently have spectroscopic data for the four additional transition objects labeled in Fig. 2. All show the clear presence of components with early-type spectra, ranging in age from $\lesssim 2 \times 10^{7}$ years to $\gtrsim 10^{8}$ years, consistent with being post-starburst populations (Canalizo \& Stockton 1998; see also Boroson \& Oke 1984). The next, and more difficult, stage in this investigation will be to try to discern and date fading starburst components in QSOs lying closer to the powerlaw line in the FIR two-color diagram to see whether these host galaxies are consistent with the Sanders et al. (1988a) evolutionary scenario.

\section{Quasars and Radio Galaxies at Higher Redshifts}

\subsection{INTRODUCTION}

In observing interactions at low redshifts, we seem mostly to be seeing encounters between mature, well formed galaxies. As we go to higher redshifts, 
deep $H S T$ images show that galaxies tend to be more ragged and irregular. Even at a relatively modest $z \sim 1$, a large percentage of galaxies show asymmetric structure and apparently are still undergoing a major part of their disk formation. Accordingly, mergers increasingly take on the nature of successive accretion of various lumps of the still forming galaxies. The distinction between merger and accretion blurs into a continuum.

When the unusual elongated morphologies of several high-redshift radio galaxies were first discovered, the initial assumption was that these structures were the result of recent mergers (e.g., Djorgovski et al. 1986). Once it was realized that the optical elongations were aligned with the radio structure (Chambers et al. 1987; McCarthy et al. 1987), this assumption no longer seemed tenable. While the final verdict on the physical mechanisms responsible for the alignment effect is not yet in, it seems clear that it is no longer safe to assume that any global distortion or large-scale transient structure necessarily means an interaction or merger. On the other hand, the ubiquity of aligned structure in high-redshift radio galaxies and quasars may tend to mask genuine tidal features or at least make their interpretation more difficult.

With these cautions in mind, I would like to discuss briefly two objects from an $H S T$ imaging program of a complete sample of $z \sim 13 \mathrm{CR}$ quasars and galaxies Susan Ridgway and I have been conducting, which we believe are likely examples of recognizable major interactions or mergers at high redshift.

\subsection{INTERACTIONS AND MERGERS AT $z \sim 1$}

\subsection{1. $3 C 280$}

We have shown our deep HST WFPC2 and ground-based images of the radio galaxy 3C 280 in Ridgway \& Stockton (1997; see also Best et al. 1997), and I refer the reader to that paper for an illustration of the morphology. Briefly, the radio core is centered on the $\mathrm{E}$ component of a very close $\left(\sim 0^{\prime \prime} 2\right)$ double $(a$ and $b)$, well aligned with the radio axis. Also aligned with the radio axis, $\sim 1^{\prime \prime} \mathrm{W}$ of the radio core, lies an object $(c)$, elongated more-or-less transversely to the radio axis. From the $\mathrm{W}$ side of $c$, an almost semicircular arc extends to the $\mathrm{N}$, proceeding $\mathrm{E}$, and terminating close to the position of $b$. The arc is strong in both the continuum and in [O II] $\lambda 3727$ emission. Its line-to-continuum ratio appears to be the same as that of $c$, for which we have found that $\sim 50 \%$ of the continuum is due to nebular thermal emission (Ridgway \& Stockton 1997, 1998). We have considered, and rejected, several possible origins for the arc, including gravitational lensing of background objects or of components of 3C 280 itself. The most likely remaining explanation is that it is a tidal tail, either from $b$ or $c$. Mi- 
hos (1995) has discussed the visibility of merger signatures, including tidal tails, in HST WFPC2 images of high redshift objects. He concludes that typical tails would be marginally visible, if at all, in an exposure similar to ours (8800 s, F622W filter). However, we not only detect the apparent tail, but easily do so in a single $1100 \mathrm{~s}$ exposure. Mihos' simulations neglect two factors that may increase the surface brightness. Firstly, they do not include star formation during the interaction. Mihos argues that simulations show that starbursts "occur predominantly in the central regions," and that "star formation in the tidal debris is generally suppressed by the interaction." However, observations show that real tidal tails are often dominated by recent star formation (Stockton 1974; Schombert et al. 1990), so the problem may lie in the limitations of the simulations. Secondly, the surface brightness of a tail lying in the illumination cone of a quasar nucleus may be enhanced by scattering and/or photoionization. The strong line emission and evidence for a large contribution from nebular thermal emission suggests that this is likely the case for the arc of $3 \mathrm{C} 280$.

\subsection{2. $3 C 190$}

Figure 4 shows our HST WFPC2 F675W and Keck Near-Infrared Camera images of the 3C 190 field. The most striking object in the HST image is the almost perfectly straight feature offset to the $\mathrm{W}$ of the quasar, reminiscent of a similar feature seen in the [O III] line image of the low-redshift quasar, 4C 37.43 (Stockton \& MacKenty 1987). In 3C 190, however, the linear feature is strongly dominated by continuum radiation. In the IR image, we see two faint linear features: one is an apparent extension of the HST feature to the NW; the other, extending to the $\mathrm{S}$, is faintly visible on the $H S T$ image as well. Together, these feature bear a remarkable similarity to the tails of the local merger NGC 7252 (Schweizer 1982).

In addition to this evidence for a major merger, there is a swarm of small objects to the $\mathrm{N}$ and $\mathrm{NE}$ of $3 \mathrm{C} 190$, all within projected distances of $\lesssim 25$ kpc. These are likely to merge shortly with the 3C 190 host galaxy, and they thus may be examples of "building blocks" in a process of continuing galaxy formation (e.g., Pascarelle et al. 1996). 3C 190 is a compact-steepspectrum (CSS) radio source. The small sizes of CSS sources may mean that they are very young FR II sources or that they are confined by unusually high gas densities near the active nucleus (e.g., Fanti et al. 1990). While total lifetimes of FR II radio sources are probably always small compared with a merger time scale, a high central gas density could well be related to merging events. We are currently obtaining deep Keck spectroscopy of components of this remarkable system. 


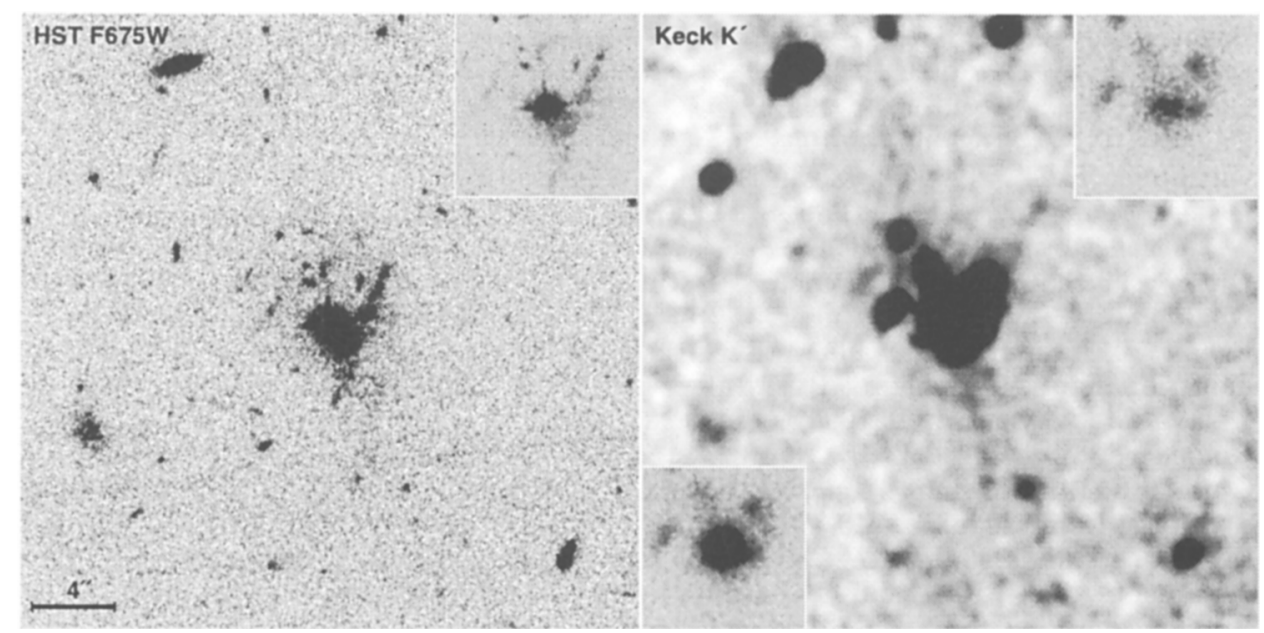

Figure 4. The field of $3 \mathrm{C} 190$. The left-hand panel shows the HST image, and the right-hand panel shows the Keck K' image, after PSF subtraction. In both panels, the insets in the upper-right corners show the same image at lower contrast; the insert in the lower-left corner of the right-hand panel shows the $\mathrm{K}^{\prime}$ image prior to PSF subtraction. The main panel $\mathrm{K}^{\prime}$ image has been smoothed slightly to bring up low-surface-brightness detail.

\section{Acknowledgements}

I thank my colleagues, Gabriela Canalizo, Laird Close, and Susan Ridgway for allowing me to present some of our observations prior to publication. Some of these results are based on observations made with the NASA/ESA Hubble Space Telescope, obtained at the Space Telescope Science Institute, which is operated by AURA, Inc., under NASA contract NAS 5-26555, and on observations made at the W.M. Keck Observatory, jointly operated by the California Institute of Technology and the University of California. Support for this work was provided by NASA through Grant No. GO05401.01-93A from the STScI, and from NSF Grant No. AST95-29078.

\section{References}

Adams, T.F. 1977, ApJS, 33, 19

Antonucci, R. 1993 ARA $\& A, \mathbf{3 1}, 473$

Bahcall, J.N., Kirhakos, S., \& Schneider, D.P. 1994, ApJL, 435, L11

Bahcall, J.N., Kirhakos, S., \& Schneider, D.P. 1995a, ApJL, 447, L1

Bahcall, J.N., Kirhakos, S., \& Schneider, D.P. 1995b, $A p J, 450,486$

Bahcall, J.N., Kirhakos, S., \& Schneider, D.P. 1996, $A p J, 457,557$

Bahcall, J.N., Kirhakos, S., Saxe, D.H., \& Schneider, D.P. 1997, ApJ, 479, 642

Barnes, J.E., \& Hernquist, L. 1991, ApJ, 370, L65

Barnes, J.E., \& Hernquist, L. 1992, ARA $\mho A$, 40, 705

Barthel, P.D. 1989, ApJ, 336, 606

Best, P.N., Longair, M.S., \& Röttgering, H.J.A. 1997, MNRAS, in press 
Bruzual A., G., \& Charlot, S. 1997, in preparation

Boroson, T., \& Oke, J.B. 1984, ApJ, 281, 535

Canalizo, G., \& Stockton, A. 1997, ApJL, 480, L5

Canalizo, G., \& Stockton, A. 1998, in preparation

Chambers, K.C., Miley, G., \& van Breugel, W. 1987, Nature, 329, 609

Disney, M.J., Boyce, P.J., Blades, J.C., Boksenberg, A., Crane, P., Deharveng, J.M., Macchetto, F., Mackay, C.D., Sparks, W.B., \& Phillipps, S. 1995, Nature, 376, 150

Djorgovski, S., Spinrad, H., Pedelty, J., Rudnick, L., \& Stockton, A. 1987, AJ, 93, 1307

Dunlop, J.S., Taylor, G.L., Hughes, D.H., \& Robson, E.I. 1993, MNRAS, 264, 455

Egami, E., Iwamuro, F., Maihara, T., Oya, S., \& Cowie, L.L. 1996, AJ, 112, 73

Fanti, R., Fanti, C, Schilizzi, R.T., Spencer, R.E., Nan, R.D., Parma, P., van Breugel, W.J.M., \& Venturi, T. 1990, $A \& A p$, 231, 333

Goldader, J., Joseph, R.D., Doyon, R., \& Sanders, D.B. 1995, ApJ, 444, 97

Gunn, J.E. 1979, in Active Galactic Nuclei, ed. C. Hazard \& S. Mitton (Cambridge Univ. Press, Cambridge), p. 213

Heckman, T.M. 1990, in IAU Colloquium 124, Paired and Interacting Galaxies, ed. J.W. Sulentic, W.C. Keel, and C. Telesco (NASA CP-3098), p. 359

Hutchings, J.B., \& Neff, S.G. 1992, AJ, 104, 1

Kim, D.-C., Sanders, D.B., Veilleux, S., Mazzarella, J.M., \& Soifer, B.T. 1995, ApJS, 98, 129

McCarthy, P.J., van Breugel, W., Spinrad, H., \& Djorgovski, S. 1987, ApJL, 321, L29

McLeod, K.K., \& Rieke, G.H. 1994, ApJ, 431, 137

McLeod, K.K., \& Rieke, G.H. 1995, ApJL, 454, L77

Mihos, J.C. $1995, A p J, 438$, L75

Mihos, J.C., \& Hernquist, L. 1996, ApJ, 464, 641

Neugebauer, G., Miley, G.K., Soifer, B.T., \& Clegg, P.E. 1986, ApJ, 308, 815

Pascarelle, S.M., Windhorst, R.A., Keel, W.C., \& Odewhan, S.C. 1996, Nature, 383, 45

Ridgway, S.E., \& Stockton, A. 1997, $A J, 114,511$

Ridgway, S.E., \& Stockton, A. 1998, in preparation

Sanders, D.B., \& Mirabel, I.F. 1996, ARA $\& A$, 34, 749

Sanders, D.B., Soifer, B.T., Elias, J.H., Madore, B.F., Matthews, K., Neugebauer, G., \& Scoville, N.Z. 1988a, ApJ, 325, 74

Sanders, D.B., Soifer, B.T., Elias, J.H., Neugebauer, G., \& Matthews, K. 1988b, ApJL, 328, L35

Scheuer, P. 1987, in Superluminal Radio Sources, ed. J. Zensus and T. Pearson (Cambridge Univ. Press, Cambridge), p. 104

Schombert, J.M., Wallin, J.F., \& Struck-Marcell, C. 1990, AJ, 99, 497

Stockton, A. 1974, $A p J, \mathbf{1 8 7}, 219$

Stockton, A. 1990. in Dynamics and Interactions of Galaxies, ed R. Wielen (SpringerVerlag, Heidelberg), p. 440

Stockton, A., Canalizo, G., \& Close, L. 1998a, in preparation

Stockton, A., \& Ridgway, S.E. 1991, $A J$, 102, 488

Stockton, A., Ridgway, S.E., \& Kellogg, M. 1998b, in preparation

Surace, J.A., Sanders, D.B., Vacca, W.D., Veilleux, S., \& Mazzarella, J.M. 1998, ApJ, 492, 116

Toomre, A., \& Toomre, J. 1972, ApJ, 178, 623

Veilleux, S., Kim, D.-C., Sanders, D.B., Mazzarella, J.M., \& Soifer, B.T. 1995, ApJS, 98, 171

Veilleux, S., Sanders, D.B., \& Kim, D.-C. 1997, $A p J, 484,92$

Voit, G.M., Weymann, R.J., Korista, K.T. 1993, $A p j$, 413, 95 